\title{
Interworking Architectures in Heterogeneous Wireless Networks: An Algorithmic Overview
}

\author{
Aderemi A. Atayero \\ Dept. of Elect. \& Info. Engrg. Covenant University, \\ Nigeria
}

\begin{abstract}
The scarce availability of spectrum and the proliferation of smartphones, social networking applications, online gaming etc., mobile network operators (MNOs) are faced with an exponential growth in packet switched data requirements on their networks. Haven invested in legacy systems (such as HSPA, WCDMA, WiMAX, Cdma2000, LTE, etc.) that have hitherto withstood the current and imminent data usage demand, future and projected usage surpass the capabilities of the evolution of these individual technologies. Hence, a more critical, cost-effective and flexible approach to provide ubiquitous coverage for the user using available spectrum is of high demand. Heterogeneous Networks make use of these legacy systems by allowing users to connect to the best network available and most importantly seamlessly handover active sessions amidst them. This paper presents a survey of interworking architectures between IMT 2000 candidate networks that employ the use of IEFT protocols such as MIP, mSCTP, HIP, MOBIKE, IKEV2 and SIP etc. to bring about this much needed capacity.
\end{abstract}

\section{General Terms}

3G Network, Wireless communication, Mobile communication

\section{Keywords}

Heterogeneous networks, HetNet, converged networks, WiFi, SIP, MIP, AIPN

\section{INTRODUCTION}

Heterogeneous Networks (a.ka. HetNets) are essentially made up of existing disparate Radio Access Network technologies used for the purposes of improving user experience, reducing RAN \& CN bottlenecks, Introducing intelligent IP traffic steering \& management, efficient load balancing and resource allocation, by ways not limited to aggregating disparate network radio resources, offloading and onloading selected or bulk PS/CS traffic between these HetNets. These disparate radio interfaces are merged both at the UE \& RAN, as a result, multi- radio frameworks(client and host based) enabling mobility and handover managements are necessary [1].

With the existing 2nd and 3rd generation technologies or services not been able to provide the required ubiquitous network coverage, high data rate or accompanying QoS levels, it's paramount that UEs makes efficient use of all available network interfaces in order to maintain an "Always Best Connected" scenario to a Corresponding Node (CN). Thus requiring multiple Radio Access Technologies (RATs) to coexist, interwork and interoperate [2, 3]. Amidst the

\author{
Elijah I. Adegoke \\ Dept. of Elect. \& Info. Engrg. Covenant University, \\ Nigeria
}

several interworking options, 3G (WiMAX/UMTS)-WIFI has been investigated beyond other inter-technology options. This is probably due to the attendant complementary offerings e.g. for WIFI: high data rates, short range, low mobility, while for 3G: relatively low data rates, long range, high mobility [4]. However, it should be noted that majority of the WiFi coverage areas could form micro cells complementing the macro cell coverage from the $3 \mathrm{G}$ BTS, thus creating an overlapping coverage area. This would definitely accommodate a longer period for VHO between $3 \mathrm{G}$ and WiFi systems since the UE can maintain connectivity with both networks. On the other hand, handover scenarios between partially/non- overlapping cells (e.g. 3G WCDMA and WiMAX) are usually characterized by short periods for the VHO. Hence, the need for buffers and fast handover processes for VHO that extend beyond the tolerance for session applications [5]. Interworking of wireless networks requires mobility management and session management across the participating RATs and the UE. The former can be classified into three: Network Layer solutions (L3), Link Layer solutions (L2) and Cross Layer solutions (L3 +L2) [6]. However, the heuristics discussed in this paper employeither MIP, its variant or SIP for mobility and session management.,

The remainder of this paper is organized as follows. The next section discusses an overview of interworking between IMT 2000 candidate networks such as WiMAX and 3G UMTS. Its subsections illustrate the different approaches employed to facilitate these interworking scenarios. discusses The paper is summarized in section 3 , and section 7 is an appendix highlighting the essential features of the literatures surveyed.

\section{INTERWORKING IN IMT 2000 NETWORKS}

\subsection{WIMAX and 3GPP Access Networks}

\subsubsection{WiMAX and $3 G P P$}

The authors of [9] propose a framework that is built around two IEEE drafts (IEEE 1900.4[10] and IEEE 802.21[7]) to perform a VHO from a $3 \mathrm{GPP}$ access network (AN) to a WiMAX AN. The IEEE 1900.4 is used to "address the architectural blocks in both the terminal and the network for effective decisions in network reconfigurability.Here the handover function is regarded as a reconfigurability enabler for networks involving multiple RATs with fixed spectrum allocation". Other enablers within the standard relating to VHO include dynamic spectrum assignment and dynamic spectrum access. The fundamental concept of the 1900.4 is to 
define a decision based system that acts on a set of actions, which are required to optimize the radio resource coordination and QoS in a heterogeneous wireless network environment [11]. The following entities within the 1900.4 are employed in the VHO, VHD and VHC; VHC $-\mathrm{t} / \mathrm{n}$, CIC $-\mathrm{t} / \mathrm{n}$, THM and NHM as proposed by the authors. These entities subscribe to the 802.21 as MIH users. Mobility management within the framework could be handled by MIP, mSCTP and SIP. Handover Initiation as discussed by the authors is based on either the IEEE 1900.4 or the IEEE 802.21.

\subsubsection{IEEE 1900.4 Handover Initiation Algorithm:}

STEP1. The CIC-t obtains MT context information and access network discovery details, which are sent to the THM and stored in a TIDB.

STEP2. Concurrently network context information is retrieved by the CIC $-n$ and forwarded to the NHM and stored in a NIDB.

STEP3. THM and the NHM then exchange context information.

STEP4. NHM "generates radio resource selection policies and constraints".

STEP5. NHM forwards them to the THM.

In a situation whereby the received policies in STEP 5 trigger a VHO, the final VHD is concluded by the terminal. However, it is essential that network policies and constraints are consistent.

\subsubsection{IEEE 802.21 Handover Initiation \\ Algorithm:}

STEP1. THM requests for link parameters from the MIHF (UE).

STEP2. MIHF (UE) responds with the requested details.

STEP3. Assuming the reported parameters indicate degrading QoS, the following occurs

STEP4. UE obtains static network information from the IEEE 802.21 MIIS.

STEP5. IEEE 802.21 MIIS forwards the requested information to the UE either in a "periodic push fashion" or a sequence of request and notification messages.

STEP6. Alternatively, the network information provided by the MIIS can as well be made available by the ANDSF.

STEP7. Based on the static information received from the MIIS or the ANDSF, the UE performs a dynamic network query by scanning the candidate networks made available by the MIIS and ANDSF. "The scanning results can be used for the identification of a potential handover target"

STEP8: UE further queries the selected target network (WiMAX) for resource availability. This can be achieved by exchanging MIH message between the THM and a MIH user within the serving PoS or between MIH users in the serving PoS and the candidate PoS. (Probably a RM).

\subsubsection{Algorithm for Handover Preparation and Execution}

STEP1. VHC-t indicates an imminent VHO by sending acommit message to the appropriate $\mathrm{MIH}$ user in its serving $\mathrm{PoS}$, which in turn forwards the request to an appropriate MIH user in the candidate PoS. (MIH users within the candidate and serving PoS could be VHC-n and/or RMs).

STEP2. UE uses the MIHF to turn on its WiMAXinterface.

STEP3. Mobility management is handled by MIPv4, such that the VHC-t activates a MIPv4 operation: UE sends a MIPv4 FA solicitation.

STEP4. WiMAX FA sends FA advertisement.

STEP5. MIPv4 registration occurs between the UE, WiMAX FA and the HA located in the PDN GW.

STEP6. HA located in the PDN GW sends registration reply via the WiMAX FA.

STEP7. Traffic flows via the WiMAX FHR through a tunnel to the PDN GW and to the CN.

In the architecture described, the UE is originally connected to a 3GPP network; WiMAX FA is located within the FHR.

\subsubsection{Mobile WiMAX (802.16) and WCDMA (3G UMTS)}

In [12], Mobile WiMAX and UTRAN are interworked based on the 3GPP EPC, an all-IP network which makes available "interworking functionalities such as UE authentication, access network discovery, QoS consistency and seamless handover" for IP based integration of non-3GPP access networks. The nodes and logical interfaces introduced into the EPC to facilitate the VHO are the: DFF, FAF and ANDSF; S2a, STa, Gx, S14, X300 and X200. The DFF and FAF are base station level logical entities located in the respective networks of the RATs involved. The former resides in the source network and its responsible for interconnectivity, data forwarding and buffering for the UE, while the later resides in the target network and its responsible for authenticating the UE in the target network prior to the VHO and enabling an IP-based handover using PMIPv6 [13]. The ANDSF is used to obtain information about neighboring cells (3GPP and non-3GPP). However, these logical entities (DFF, FAF \&ANDSF) described do not provide any form of radio connection to the UE except for IP tunnel communications.

The functionalities of the logical interfaces introduced are: $S 2 a$-Interface between the WiMAX ASN-GW and the EPC P-GW. It supports terminal mobility towards the P-GW.

Sta -Interface between the WiMAX ASN-GW and the AAA/HSS.It isused for authenticating the UE.

$G x$-Interface between the WiMAX ASN-GW and the PCRF.It is used for QoS consistency and policy charging. S14 -Interface between UE and ANDSF.

$X 200$ - IP tunnel between FAF and UE.

$X 300$ - IP tunnel between DFF and UE.

VHO across the two networks might be initiated by link layer triggers or policy management implemented by a MNO for a subscriber. The DFF and FAF assume specific functionalities of the SN and DN, i.e. for a VHO between mobile WiMAX and UTRAN (left to right), the DFFwould comprise of logical 
functionalities and protocols associated to the WiMAX BTS, while the FAF emulates the UTRAN RNC with the appropriate protocols and functionalities. The discovery of the DFF and FAF is facilitated by the ANDSF. This eliminates the need for the UEs to actively scan for available/neighbor RANs, thus reducing interference and increasing battery life on the UE. The VHO process described is summarized in the following algorithm.

\subsubsection{Algorithm for Vertical Handover:}

STEP1. UE discovers the ANDSF so as to obtain target 3GPP UTRAN information

STEP2. UE obtains DFF \& FAF addresses from ANDSF.

STEP3. UE performs cell measurement on the available 3GPP cells and selects a target cell.

STEP4. UE establishes IP tunnel with the FAF, attaches and authenticates with the EPC via the FAF.

STEP5. FAF performs authentication with the UTRAN on behalf of the UE.

STEP6. UE instructs the FAF to relocate its session to the target 3GPP cell.

STEP7. FAF initiates resource reservation and relocates UE to the target network via a handover command.

STEP8. UE prepares to detach from the WiMAX network by sending a relocation request and instructing DFF to initiate data forwarding.

STEP9. DFF establishes tunnel to the target FAF (IP of FAF is obtainable in step 8: data forwarding request)

STEP10. DFF performs handover between the WiMAX BTS and DFF on behalf of the UE. At this instant, the logical connection(s) between the UE and (DFF/FAF) and is lost.

STEP11. Source network buffers data destined for the UE and forwards them to the DFF.

STEP12. DFF initiates data forwarding with the FAF by sending a data forwarding indication to the FAF.

STEP13. DFF forwards buffered packets to the FAF. ("Note that the FAF starts to buffer the user data after transmitting Handover Command in step 7"). During this period, the UE moves to the target 3GPP UTRAN cell during the DFF forwarding period.

STEP14. DFF starts deregistration process after data forwarding is completed

STEP15. UE initiates PDP context based on the QoS in use in the WiMAX network.

STEP16. UE resumes data communication via the 3GPP UTRAN cell.

Since the UE is connected to the same P-GW before and after the VHO, the PMIPv6 [13] tunnel in the P-GW is switched from ASN-GW to S-GW, the S-GW would send a binding update to the P-GW [14].. We note here that steps 1 to 8 occur while the UE is still in the source network, thus significantly reducing the active VHO time.

\subsubsection{WiMAX and 3G UMTS-IMS Based}

[15] Describes a similar VHO scenario (WiMAX-3G cellular network: right to left) from a centralized IMS-MIP perspective. The IMS is responsible for session mobility, while MIP (v4 or v6) is used for mobility management across both networks $[16,17]$. However, MIPv4 was adopted for the proposed architecture, which comprises of four disparate RATs (WiMAX, 3G UMTS, CDMA 2000 and WiFi), with individual MIP FA/HA and local P-CSCF located within the corresponding GWs. These RATs are connected to an ALL-IP network via their corresponding GWs (ASN GW, GGSN, PDSN and (GIF)/SGSN emulator). The MIP HA, I and S$\mathrm{CSCF}$ are located in the home network. The session handover process is as given in the following algorithm:

\subsubsection{Session Handover Algorithm}

STEP1. MN attaches to the UMTS network, performs the PDP context activation and service registration as described in [18].

STEP2. GGSN (MIP FA) sends agent advertisement

STEP3. MN acquires IP address and proceeds with MIPv4 registration with the MIP HA.

STEP4. MIP HA responds to the MIPv4 registration message.

Thereafter, the MN proceeds with establishing a SIP session with the corresponding node $(\mathrm{CN})$.

STEP5. MN SIP-UAC sends a SIP "invite" message to the corresponding node via the $\mathrm{P}-\mathrm{CSCGF}$. The invite message sent also requests for information regarding the precondition QoS requirements associated with initiating the session.

STEP6. CN replies with a "183 SESSION PROGRESS" message. This message also contains a SDP answer.

STEP7. MN sends a precondition acknowledgement "PRACK" to the CN.

STEP8. CN replies the MN with a "200 OK" SIP message.

STEP9. MN sends UPDATE message to the $\mathrm{CN}$ verifying resource reservation.

STEP10. CN replies MN with a "200 OK" SIP message.

STEP11. MN replies with "ACK" SIP message via the same path. At this instant, the session between the $\mathrm{CN}$ and $\mathrm{MN}$ is in progress via the UMTS network.

STEP12. The MN roams into a WiMAX network, thereby triggering a session handover to the WiMAX network.

STEP13. MN performs link layer registrations with the WIMX network.

In STEPs 11- 13, MN performs MIPv4 registration with the WiMAX network (similar to steps 1-3)

STEP14. Binding update occurs between $\mathrm{MN}$ and $\mathrm{CN}$ to avoid triangular routing.

STEP15. MN sends SIP "Re-INVITE" message (using the same CALL-ID and other old connection identifiers) to the SIP UAS in the CN.

STEP16. Repeat STEP 5 to STEP 10: At this instant, the session between the $\mathrm{CN}$ and $\mathrm{MN}$ is in progress via the WiMAX network.

STEP17. MN drops the data pipeline with the UMTS network by sending a SIP "BYE" message.

${ }^{1}$ The authors further modeled the performance of the VHO and also presented an accompanying analytical and numerical analysis.

\footnotetext{
${ }^{1}$ It is assumed that both the WiMAX and 3 G Network interconnect to a central IP network.
} 


\subsubsection{WiMAX and $3 G$ UMTS-Non-overlapping IOverlapping Handover}

In [19] a theoretical model to interworking of RATs (WiMAX \& UMTS) is postulated by employing an intelligent user terminal capable of sustaining dual radio links (WiMAX \& UMTS), processing, monitoring, comparing received signals and implementing inter-technology handover. The architecture employs MIPv4 [16] at L3 and SIP [8] for handling session management and negotiations at the upper layer (L7) of the OSI model. Two handover scenarios (overlapping and non-overlapping) are described in this technical paper. 2.1.4.1 Algorithm for Non-overlapping

\section{Handover}

The non-overlapping handover process can be summarized into the following 15 steps:

STEP1: UE connects to a UMTS network and performs Link Layer Registrations. UE performs service registration to the UMTS network by sending a SIP INVITE message to the UMTS P-CSCF.

STEP2: UMTS P-CSCF sends "ACK" message to the UE.

STEP3: UE sends "OK" message to the UMTS P-CSCF and data transfer commences via the UMTS network.

STEP4: As the UE approaches the cell edge of another network, it begins to receive FA CoA Advertisements from the target network (WiMAX). UE monitors RSL of source and target network, UE makes intelligent decision to handover. After a positive VHD, UE begins WiMAX Link layer registration.

STEP5-6: UE successfully registers its FA CoA with HA (MIP registration: MIP Message request to HA \& MIP respond notification from HA.)

STEP7: UE initiates service handover using the SIP REINVITE message (with the same user ID \& QoS) to the WiMAX P-CSCF.

STEP8: application server (AS) via the WiMAX P-CSCF sends SDP message to the UE

STEP9: UE sends SDK ACK to the AS

STEP10: AS sends SDP reply "OK" to the UE

STEP11: UE sends SDP "UPDATE" message to the AS.

STEP12: AS updates UE information and sends "OK" message to UE.

STEP 13: UE sends SDP ACK message to the AS. HA stops sending packets to the UE, HA forwards packets to WiMAX FA, WiMAX FA forwards 1st packet to UE. UE performs binding update with the HA, CN \& WiMAX ASN-GW: FA)

STEP 14: UE sends "BYE" notification message and the UMTS P-CSCF

STEP 15: UMTS P-CSCF sends "OK" message to UE and UMTS network pipe is torn down.

\subsubsection{Algorithm for Overlapping Handover.}

On the other hand, the overlapping VHO process differs from the former and can be summarized into the following 15 steps as well.

STEP1: UE roams into an overlapping network coverage area, UE performs a dual link layer registration with both UMTS and WiMAX networks, during which the UE receives regular updates from both FAs' residing in the corresponding networks.
STEP2: UE eventually locks onto the RAT with the best RF parameters (It's assumed the UE locks to the UMTS network). The UE performs service registration to the UMTS network by sending a SIP INVITE message to the UMTS P-CSCF. STEP 3: UMTS P-CSCF sends "ACK" message to the UE. STEP 4: UE sends "OK" message to the UMTS P-CSCF and data transfer commences via the UMTS network and completes its service registration. UE monitors RSL of source and target network, UE makes intelligent decision to handover. After a positive VHD, UE begins WiMAX Link layer registration.

STEP 5-6: UE successfully registers its FA CoA with HA (MIP registration: MIP Message request to HA \& MIP respond notification from $\mathrm{HA}$.)

STEPS 7-13(same as non-overlapping algorithm): UE performs Service Handover and binding update.

STEP 14: UE sends "BYE" notification message and the UMTS P-CSCF

STEP 15: UMTS P-CSCF sends "OK" message to UE and UMTS network pipe is torn down.

The paper also highlights latency results for UMTS-WiMAX handover and vice versa with simulated traffic (VoIP \& FTP transfers).

\subsubsection{IEEE 802.16e and 3GPP UTRAN-WAG Approach}

[5] Also proposed an interworking system for IEEE 802.16e and 3GPP UTRAN wireless networks systems. The interworking RAT proposition was guided by the need to implement little or no change to the existing underlying core network infrastructure of the participating RATs and to provide a practicable and temporal interworking solution. In order to abstract the lower layer dissimilarities of the RATs involved, MIP [16] was employed as an interconnection protocol. The proposed architecture links the WiMAX ASN with the 3GPP network using a WAG which is responsible for filtering and enforcing routing of packet to/from the PDN. It also adopts a PDN hosted HA that manages the FAs of the participating RATs. VHO in this context requires a UE (capable of connecting to either of the participating RATs). The handover call flow process for WiMAX to 3GPP can be outlined in the following 13 steps $^{2}$.

\subsubsection{Call Flow Algorithm for WiMAX - 3GPP UTRAN Handover.}

STEP1: WiMAX BTS sends periodic information (such as carrier frequency, scrambling code etc.) of neighboring cells WiMAX \& 3GPP to the UE. It is assumed that there is an existing collaboration between the 3GPP and WiMAX networks, which would allow either RAT to send information about the other. This task can also be performed by the UE.

\footnotetext{
2 Before the VHO WiMAX-3GPP \& (3GPP- WiMAX)

- UE is connected to the WiMAX AN (3GPP) Network,

-UE enters overlapping zone and measures radio parameters of available neighboring 3 GPP (WiMAX BTS) cells.

In the case of an event triggered VHO. Triggered events could be degrading RSL, higher QoS support \& cost
} 
STEP2: Based on the information received in Step 1, UE measures the signal level, quality and other relevant (as configured) parameters of the available neighboring cells.

STEP3: UE sends the measurement report to the WiMAX BTS, which forwards it to the ASN-GW

STEP4: BTS/ASN-GW initiates a Handover (HO) request message via the WAG \& PDG to the target 3GPP site. PDG performs a DNS request in order to get the address details for the soon to be GGSNs servicing the target 3GPP site. PDG selects and forwards the $\mathrm{HO}$ request to one of the servicing GGSNs. Should the selected GGSN fail to respond to the HO request, the PDG would send the request to another GGSN from the resolution list.

STEP5: The GGSN (that responded to the HO request) relays the HO request to the servicing SGSN(s) of the target NodeB STEP6: The target RAN reserves bearer and radio resources for the $\mathrm{HO}$ request. It also checks to see if it can accommodate the incoming $\mathrm{HO}$ request as well as the requested QoS support.

STEP7: After successfully reserving resources, the NodeBs that support the handover send a HO support message to the WiMAX ASN GW with a handover decision function.

STEP8: Haven received multiple results from the NodeBs available to support the VHO, the ASN-GW decides on the best fit to handle the VHO. ASN-GW sends HO command message alongside connection setup information to the UE. In order to reduce the size of $\mathrm{HO}$ command message and reduce latency, a predefined radio configuration mechanism, downloadable to the UE is proposed by the author.

STEP9: ASN-GW forwards HO confirmation and selected target NodeB identification information to the PDG. At this instant, the WiMAX network resources are released.

STEP10: Upon receipt of the HO confirmation, the PDG would send an MIP update to the HA informing it about the UEs network movement. HA seizes to forward packets destined for the UE via the PDG/FA and buffers the incoming packets till it receives an MIP update from the target NodeB.

STEP11: UE attaches to the 3GPP network. This involves, accessing the SGSN, authentication and location update.

STEP12: UE initiates PDP context, while furnishing the serving GGSN with its global home address (assigned by HA or an external entity)

STEP13: UE connects with GGSN (which also hosts the MIP FA for the 3GPP Network) performs MIP registration/Update with the HA (using the global home address and the CoA from the GGSN/FA). Buffered packets are now delivered to the UE via the NodeB.

Also discussed in this white paper is the reverse VHO call flow process.

\subsubsection{Call Flow Algorithm for 3GPP UTRAN - WIMAX Handover}

Below is the handover call flow process for 3GPP to WiMAX, which can be outlined in the following 15 steps:

STEP1: 3GPP UTRAN detects need for VHO, sends measurement control message (containing details of neighboring WiMAX cells) to the UE. The message is sent in a compressed mode because the UE is currently in a FDD spectrum. In order to perform measurements on a WiMAX (TDD spectrum) preamble frame, it must switch into a compressed mode.

STEP2: UE measures the signal level, quality and other relevant (as configured) parameters of the available neighboring WiMAX cells

STEP3: UE sends the measurement report to the NodeB, which forwards it to the RNC.

STEP4: RNC initiates a Handover (HO) request message to notify the target WiMAX cell of an imminent VHO. The HO request message typically contains information such as the UE APN, candidate BTS identifiers, required QoS etc. are sent via the SGSN to the GGSN/FA. Upon receiving the message, GGSN/FA performs a DNS request in order to get the address details for the PDGs servicing the UE's soon to be APN (WiMAX cell). GGSN/FA selects and forwards the HO request to one of the servicing PDGs from the DNS resolution list, if the selected PDG fails to respond to the HO request, the GGSN/FA would send the request to another PDG contained in the resolution list. The HO request message is further sent through the PDG, WAG, WiMAX HA, and ASN GW/FA down to the WiMAX BTS. The RAN also checks to see if it (WiMAX AN) can accommodate the incoming $\mathrm{HO}$ request as well as the requested QoS.

STEP5: WiMAX BTSs, that can support the request, forwards a HO support message to the RNC.

STEP6: RNC decides on the best fit cell to handle the VHO and sends $\mathrm{HO}$ command message alongside connection setup information to the UE.

STEP7: RNC forwards HO confirmation and selected target WiMAX cell identification information to the GGSN/FA. At this instant, the 3GPP network resources are released

STEP8: Upon receipt of the HO confirmation, the GGSN/FA (which also hosts the MIP FA for the 3GPP Network) would send an MIP update to the HA informing it about the UEs network movement. Then the HA seizes to forward packets destined for the UE via the GGSN/FA and buffers the incoming packets till it receives an MIP update from the target WiMAX cell.

STEP9: In order to reduce the latency associated with the $\mathrm{VHO}$, the information contained in the HO request message earlier sent should require the WiMAX BTS to provide a noncontention based initial ranging option for the UE. This is made possible by including a Fast Ranging Information Element in the UL MAP contained in the WiMAX Frame. Else, the UE would have to undergo the normal ranging procedure, which is OK for NRT applications.

STEP10: UE commences connection setup via the Ranging request command and response notification with the WiMAX BTS.

STEP11: UE requests and obtains a local IPv4 from the WiMAX DHCP server. (For IPv6 scenarios, the link local addresses can be used. i.e. UE doesn't perform DHCP request.)

STEP12: UE performs MIP registration with the ASN GW/FA and the WiMAX HA, in order to associate its CoA and its local address. 
STEP13: UE initiates a DNS resolution for serving PDGs addresses using its APN to the DNS server via the ASN - GW. The APN is used to specify the requested network services. Resolution response is sent via the same path, and UE selects a PDG from the DNS response list. (PDG selected might not necessarily be the same as that selected by the GGSN/FA in STEP4)

STEP14: UE sets up a tunnel with the selected PDG using IKEv2 [20], and informs it about its local (CoA) and global home address. Each time the visiting UE changes ASN GW, it has to obtain a new local IP address, i.e. set up a new IPSec tunnel. In order to reduce the associated latency with such Inter-WiMAX mobility scenarios, MOBIKE [21] could be used as against "tearing down existing security associations during a layer 3 Handoff' [22].

STEP15: WiMAX HA alongside PDG initiate a MIP registration with the $\mathrm{HA}$, and buffered packets are now delivered to the UE via the WiMAX Access Network.

\section{SUMMARY}

With interworking of RATs in place, MNOs can offer the same services and features to subscribers irrespective of the access network used. It is expected that inter-technology mobility will afford operators additional means of maximizing the productivity of already deployed access networks, thereby furnishing tools for the satisfaction of application requirements. Inter- technology mobility provides an avenue for the rationalization of existing portfolios of operators with multiple access networks, as well as reduces the ROI time on deployment of new applications. A general characteristic of NG networks will be seamless integration of communication networks, in which air interfaces will be capable of intelligently connecting people and devices over various networks of different complexities. We have presented in this paper a comprehensive overview of various means of achieving this interconnectivity between different types of networks.

\section{REFERENCES}

[1] Oliver Blume et al "A Generic Signalling Framework for Seamless Mobility in Heterogeneous Wireless Networks http://arxiv.org/ftp/arxiv/papers/1105/1105.1516.pdf

[2] Ian F. Akyildiz And Shantidev Mohanty, "A Ubiquitous Mobile Communication Architecture for NextGeneration Heterogeneous Wireless Systems" IEEE Radio Communications • June 2005

[3] Ken Murray, Rajiv Mathur \& Dirk Pesch, "Network Access and Handover Control in Heterogeneous Wireless Networks for Smart Space Environments". $1^{\text {st }}$ International Workshop on Managing Ubiquitous Communications and Services (MUCS).

[4] J. P. Chen, W. S. Chen, J. Y. Chen, H. T. Chu, and L. Hsieh "Signalling analysis of SIP-based terminal mobility protocol between WLAN and WMAN, Communication Systems and Network", 2007.

[5] Venkat Annadata Tech Mahindra (R\&D) Services Ltd. 802.16e \& 3GPP Systems Network Handover Interworking,
[6] Ian F. Akyildiz, Jiang Xie, And Shantidev Mohanty “A Survey Of Mobility Management In Next-Generation All-Ip-Based Wireless Systems". IEEE Wireless Communications. August 2004

[7] IEEE 802.21-2008, "IEEE Standard for Local and Metropolitan Area Networks - Media Independent Handover Services,"

http://standards.ieee.org/findstds/standard/802.212008.html

[8] Rosenberg, et. al.” SIP: Session Initiation Protocol” RFC 3261, June 2002

[9] Lambros Sarakis et al "Seamless Service Provision for Multi Heterogeneous Access", IEEE Wireless Communications. October 2009

[10] IEEE 1900.4-2009, "IEEE Standard for Architectural Building Blocks Enabling Network-Device Distributed Decision Making for Optimized Radio Resource Usage in Heterogeneous Wireless Access Networks," http://standards.ieee.org/findstds/standard/1900.42009.html

[11] Soodesh Buljore et al, " Architecture and Enablers for Optimized Radio Resource Usage in Heterogeneous Wireless Access Networks: The IEEE 1900.4 Working Group”, IEEE Communications Magazine. January 2009

[12] William J. Song et al "Improvements to Seamless Vertical Handover between Mobile WiMAX and 3GPP UTRAN through the Evolved Packet Core" IEEE Communications Magazine • April 2009

[13] S. Gundavelli et al., "Proxy Mobile IPv6," RFC 5213, Aug. 2008.

[14] P. Taaghol, A. K. Salkintzis, and J. Iyer, "Seamless Integration of Mobile WiMAX in 3GPP Networks," IEEE Communications. Mag., vol. 46, no. 10, Oct. 2008

[15] Kumudu S. Munasinghe, Abbas Jamalipour "Interworked WiMAX-3G Cellular Data Networks: An Architecture for Mobility Management and Performance Evaluation

[16] C Perkins "IP Mobility Support for IPv4, Revised rfc5944"

[17] C Perkins, D. Johnson \& J. Arkko "Mobility Support in IPv6 rfc 6275"

[18] Kumudu S. Munasinghe, Abbas Jamalipour and Branka Vucetic "Interworking between WLAN and 3G Cellular Networks An IMS Based Architecture"

[19] M. M. A. Khan, M. F. B. Ismail and K Dimyati "Interworking between WiMAX and UMTS to provide seamless services" April, 2010. International Journal of the Physical Sciences Vol. 5(6), pp. 852-864, June, 2010

[20] C. Kaufman (Ed), Internet Key Exchange (IKEv2) Protocol, RFC 4306, December 2005

[21] P. Eronen (Ed) “MOBIKE”, RFC 4555, June 2006, "IKEv2 Mobility and Multihoming Protocol

[22] Ashutosh Dutta. "Systems Optimization for Mobility Management”. PhD Thesis. 2010 @ Columbia University. 


\section{ACRONYMS}

ACK- acknowledgement

AN - Access Network

ANDSF -Automatic Network Discovery and Selection

Function

APN - Access Point Name

AR- Access Router

ASN GW - Access Service Network Gateway

AU-IG - Authentication Centre -Interworking Gateway

CIC- Context Information Collector (Terminal / Network)

$\mathrm{CN}$-Core Network

CoA - Care of Address

CS - Circuit Switch

DFF - Data Forwarding Function

FA-Foreign Agent

FAF -Forward Attachment Function

FHR - First Hop Router

GS- Gateway Station

HA -Home Agent

HO- Handover

HIP -Host Identity Protocol

IMS -IP Multimedia System

LCoA -Local Care of Address

MAP- Mobility Anchor Point

MICS - Media-independent command service

MIES - Media-independent event service

MIH -Media Independent Handover

MIHF - Media Independent Handover Function

MIIS- Media-independent information service

MIP - Mobile IP

MPA -Media Pre-authentication

MT- Mobile Terminal

NHM- Network Handover Manager

NIDB- Network Information Handover

NIA- Network Interworking Agent ( $3^{\text {rd }}$ party)

NIG- Network Interworking Gateway

P-CSCF -Proxy -Call Session Control Function

PDN GW- Packet Data Network Gateway

PDSN - Packet Data Serving Node

PHT - Proactive handover tunnel

PoS -Point of Service

PS - Packet Switch

RAN - Radio Access Network

RAT - Radio Access Technology

RCoA - Regional Care of Address

$\mathrm{RM}$ - Resource Manager

RNC - Radio Network Controller

SB -Switch Bank

S-CSCF -Serving -Call Session Control Function

SDP - Session Description Protocol

THM - Terminal Handover Manger

TIDB- Terminal Information DB

UAC - User Agent Client

UE- User Equipment

UTRAN - Universal Terrestrial Radio Access Network

VHC- Vertical Handover Control

VHD- Vertical Handover Decision

VHO - Vertical Handover

WAG -Wireless Access Gateway

WiMAX - Worldwide Interoperable Microwave Access 